11

\title{
Генерация широкополосного хаотического излучения в гиротронах в режиме перекрытия высокочастотного и низкочастотного резонансов
}

\author{
(C) Р.М. Розенталь, ${ }^{1}$ Н.С. Гинзбург, ${ }^{1,}$ А.С. Сергеев, ${ }^{1}$ И.В. Зотова, ${ }^{1}$ А.Э. Федотов, ${ }^{1}$ В.П. Тараканов ${ }^{2,3}$ \\ ${ }^{1}$ Институт прикладной физики РАН, \\ 603950 Нижний Новгород, Россия \\ ${ }^{2}$ Национальный исследовательский ядерный университет „МИФИ“, \\ 115409 Москва, Россия \\ ${ }^{3}$ Объединенный институт высоких температур РАН, \\ 125412 Москва, Россия \\ ฯ e-mail: ginzburg@appl.sci-nnov.ru
}

(Поступило в Редакцию 19 декабря 2016 г.)

Показана возможность значительного (до 10\%) расширения полосы шумоподобного излучения в гиротронах за счет специальной настройки гирочастоты относительно критической частоты рабочей моды, при которой для заданного значения поступательных и поперечных скоростей электронов достаточно сильно разнесены высокочастотный и низкочастотный циклотронные резонансы. При больших превышениях над порогом возникает перекрытие полос генерации на указанных резонансах. Принципиальным моментом для корректного анализа режимов широкополосной генерации в рамках усредненного подхода является учет конечности времени пролета электронов через пространство взаимодействия и соответственно учет наклона дисперсионной характеристики электронного пучка относительно дисперсионной характеристики волны. Полученные результаты подтверждены посредством прямого трехмерного PIC (particle-in-cell) моделирования хаотических режимов генерации гиротрона $8 \mathrm{~mm}$ диапазона длин волн.

DOI: 10.21883/JTF.2017.10.45001.2134

\section{Введение}

Гиротроны являются наиболее мощными источниками коротковолнового электромагнитного излучения, круг приложений которых постоянно увеличивается [1-3]. При этом для ряда практических задач, например, для нагрева плазмы [4], тестирования электропрочности многочастотных волноведущих трактов [5], специальных локационных приложений [6] представляет интерес генерация предельно широкополосного излучения. Расширение полосы излучения гиротронов может быть достигнуто за счет использования автомодуляционных режимов генерации, реализующихся при значительном превышении тока над порогом [7]. Подобные режимы неоднократно наблюдались экспериментально [8-10], в частности, при установке дополнительных отражателей (диафрагм) в выходной тракт гиротрона [11-13], что позволяет снизить бифуркационные значения токов. Следует отметить, однако, что относительная ширина спектра генерации в этих экспериментах, измеряемая по принятому (см. $[14,15])$ в теории широкополосных сигналов уровню $-10 \mathrm{~dB}$, не превышала $2 \%$.

В настоящей работе показана возможность значительного расширения полосы шумоподобной генерации гиротронов до величины порядка 10\%. Для этого необходима специальная настройка гирочастоты (величины магнитного поля) относительно критической частоты рабочей моды $\omega_{c}$, при которой для заданного значения поступательной скорости электронов достаточно сильно разнесены высокочастотный и низкочастотный циклотронные резонансы, возникающие при пересечении дисперсионных характеристик волноводной моды $h=c^{-1} \sqrt{\omega^{2}-\omega_{c}^{2}}$ и электронного потока $\omega-h V_{\| 0}=\omega_{H}$ (где $\omega-$ частота, $h$ - продольное волновое число, $\omega_{H}$ - гирочастота). В этом случае при умеренном превышении тока пучка над порогом в спектре генерации будут наблюдаться две разнесенные спектральные полосы. Однако увеличение инжектируемого тока при оптимально подобранных параметрах гиротрона приводит к перекрытию полос и генерации сверхширокополосного излучения.

Важно подчеркнуть, что принципиальным моментом для анализа указанных режимов в рамках усредненного подхода является учет конечности времени пролета электронов через пространство взаимодействия [16]. В большинстве предшествующих работ, посвященных динамике нестационарных процессов в гиротронах, в которых эволюция поля описывается параболическим уравнением, предполагалось, что время пролета пренебрежимо мало в масштабе времени изменения амплитуды поля [17-19]. В таком предположении линия пучка на дисперсионной диаграмме проходит вертикально, что не позволяет корректно описать исследованные далее режимы двойного пересечения. Результаты, полученные в рамках усредненной самосогласованной модели, подтверждены на основе прямого PIC (particlein-cell) моделирования хаотических режимов генерации гиротрона $8 \mathrm{~mm}$ диапазона длин волн с использованием трехмерной версии кода KARAT [20]. 


\section{1. Модель и основные уравнения}

Предположим, что пространство взаимодействия гиротрона представляет собой отрезок цилиндрического волновода, в котором $T E_{m, n}$-волна возбуждается на квазикритической частоте $\omega_{c}$. Одновременно частота излучения $\omega$ близка к гирочастоте $\omega_{H}=e H_{0} / m c \gamma$. В таких условиях, выбирая в качестве несущей частоту отсечки, представим поле в рабочем пространстве в виде

$$
\mathbf{E}=\operatorname{Re}\left(A(z, t) \mathbf{E}_{\perp}(r) \exp \left(i \omega_{c} t-i m \phi\right)\right),
$$

где $A(z, t)$ - медленно меняющаяся комплексная амплитуда поля, функция $\mathbf{E}_{\perp}(r)$ описывает радиальную структуру рабочей моды, $\varphi$ - азимутальный угол. Электронно-волновое взаимодействие может быть описано системой уравнений, включающих в себя неоднородное уравнение параболического типа для эволюции комплексной амплитуды поля совместно с усредненными уравнениями движения электронов [16]:

$$
\begin{gathered}
i \frac{\partial^{2} a}{\partial Z^{2}}+\frac{\partial a}{\partial \tau}=\frac{I_{0}}{2 \pi} \int_{0}^{2 \pi} p d \theta_{0} \\
\frac{\partial p}{\partial Z}+\frac{g^{2}}{4} \frac{\partial p}{\partial \tau}+i p\left(\Delta-1+|p|^{2}\right)=-a .
\end{gathered}
$$

В системе уравнений (2),(3) использованы следующие нормированные переменные:

$$
\begin{gathered}
\tau=\frac{\omega_{c} t \beta_{\perp 0}^{4}}{8 \beta_{\| 0}^{2}}, \quad Z=\frac{\beta_{\perp 0}^{2} \omega_{c} z}{2 \beta_{\| 0} c}, \\
p=\frac{\left(p_{x}+i p_{y}\right) e^{-i \omega_{c} t+i(m-1) \phi}}{p_{\perp 0}}, \\
a=\frac{e A J_{m-s}\left(R_{0} \omega_{c} / c\right)}{m c \omega_{c} \gamma_{0} \beta_{\perp 0}^{3}}, \\
I_{0}=16 \frac{e I_{b}}{m c^{3}} \frac{\beta_{\| 0}}{\beta_{\perp 0}^{6} \gamma_{0}} \frac{J_{m-1}^{2}\left(R_{0} \omega_{c} / c\right)}{\left(v_{p}^{2}-m^{2}\right) J_{m}^{2}\left(v_{p}\right)},
\end{gathered}
$$

где $I_{b}-$ ток электронного пучка, $J_{m}-$ функция Бесселя, $v_{p}-p$-й корень уравнения $J_{m}^{\prime}(v)=0, R_{0}-$ радиус инжекции винтового электронного пучка, $g=$ $=\beta_{\perp 0} / \beta_{\| 0}-$ питч-фактор, $V_{\perp 0}=\beta_{\perp 0} c$ и $V_{\| 0}=\beta_{\| 0} c-$ начальные значения поперечной и продольной скоростей электронов, $\Delta=2\left(\omega_{c}-\omega_{H}\right) / \omega_{c} \beta_{\perp 0}^{2}-$ параметр расстройки между критической частотой рабочей моды и невозмущенным значением гирочастоты.

Будем считать, что на входе в пространство взаимодействия электроны равномерно распределены по фазам циклотронного вращения $p(Z=0)=\exp \left(i \theta_{0}\right)$, $\theta_{0} \in[0,2 \pi)$. С катодного конца пространство взаимодействия ограничено закритическим сужением: $a(0, \tau)=0$. В то же время на выходе системы в сечении $Z=L$

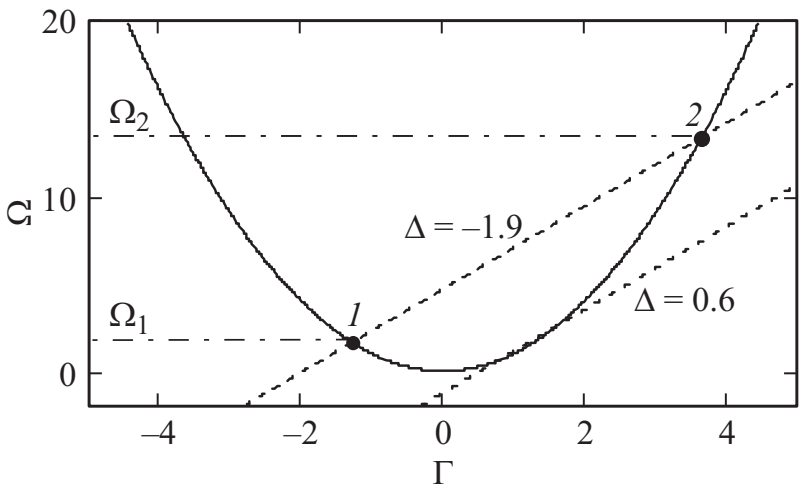

Рис. 1. Дисперсионные диаграммы волноводной моды (сплошная линия) и электронного пучка (пунктирные линии) при заданном питч-факторе $g=1.3$ и различных значениях расстройки циклотронного резонанса: $\Delta=0.6$ соответствует режиму касания, когда имеет место взаимодействие только с попутной волной; $\Delta=-1.9$ соответствует режиму пересечения, когда имеет место одновременное взаимодействие с обратной (точка 1) и попутной (точка 2) волнами.

(где $L=\beta_{\perp 0}^{2} \omega_{c} l / 2 \beta_{\| 0} c-$ нормированная длина резонатора) будем использовать излучательное граничное условие [7]

$$
a(L, \tau)+\frac{1}{\sqrt{\pi i}} \int_{0}^{\tau} \frac{1}{\sqrt{\tau-\tau^{\prime}}} \frac{\partial a\left(L, \tau^{\prime}\right)}{\partial Z} d \tau^{\prime}=0 .
$$

В использованных нормировках электронный КПД запишется в виде

$$
\eta=\frac{g^{2}}{1+g^{2}} \eta_{\perp}, \quad \eta_{\perp}=1-\frac{1}{2 \pi} \int_{0}^{2 \pi}|p|^{2} d \theta_{0},
$$

где $\eta_{\perp}$ - так называемый поперечный КПД. Заметим здесь, что для рассматриваемой системы максимальное значение поперечного КПД в стационарном режиме генерации $\eta_{\perp} \approx 0.76$ достигается при значении нормированной длины $L=15$, параметре тока $I_{0}=0.01$ и параметре расстройки $\Delta=0.61$.

Представляя амплитуду поля и поперечный импульс электронов в уравнениях (2), (3) в виде $a, p \sim \exp (i \Omega \tau-i \Gamma Z)$, где

$$
\Omega=\frac{\omega-\omega_{c}}{\omega_{c}} \frac{8 \beta_{\| 0}^{2}}{\beta_{\perp 0}^{4}}, \quad \Gamma=\frac{2 \beta_{\| 0} c}{\beta_{\perp 0}^{2} \omega_{c}} h,
$$

нормированный сдвиг частоты генерации от критической частоты и нормированное продольное волновое число, в пренебрежении связью между частицами и излучением, получим дисперсионные характеристики для электромагнитной волны

$$
\Omega=\Gamma^{2},
$$

и электронного пучка

$$
\Omega=\frac{4}{g^{2}}(\Gamma-\Delta)
$$




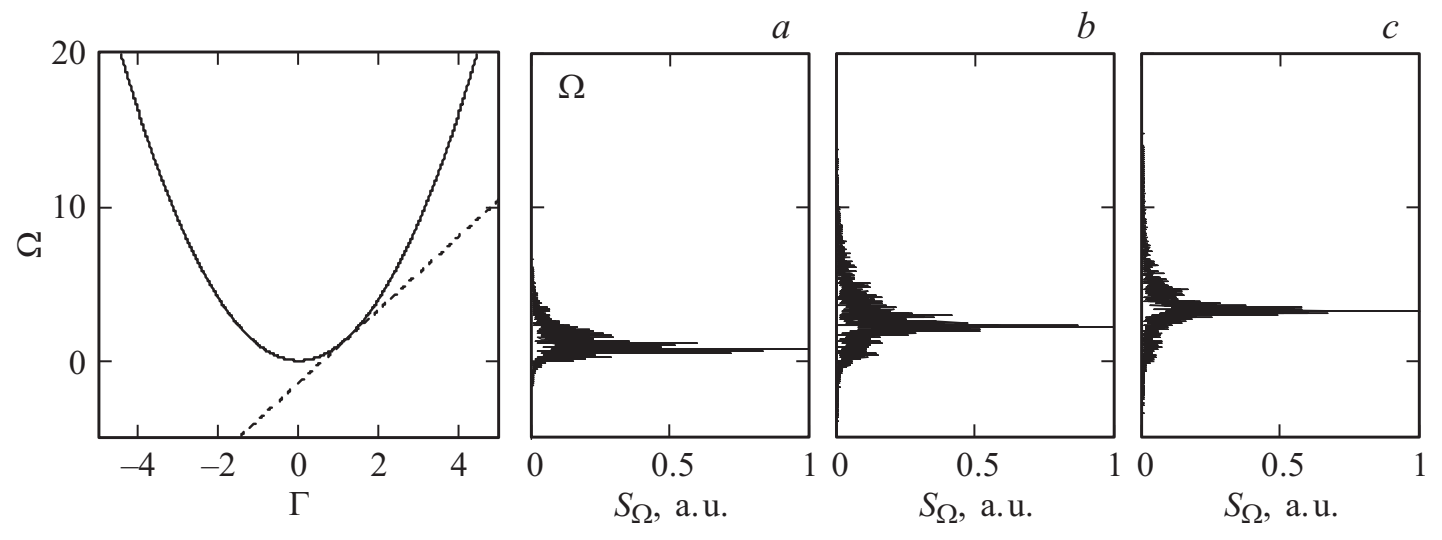

Рис. 2. Дисперсионные характеристики волны и пучка в режиме касания при $\Delta=0.6$ (слева) и спектры выходного сигнала при увеличении параметра тока, $I_{0}: a-1.0, b-5.0, c-10.0$.
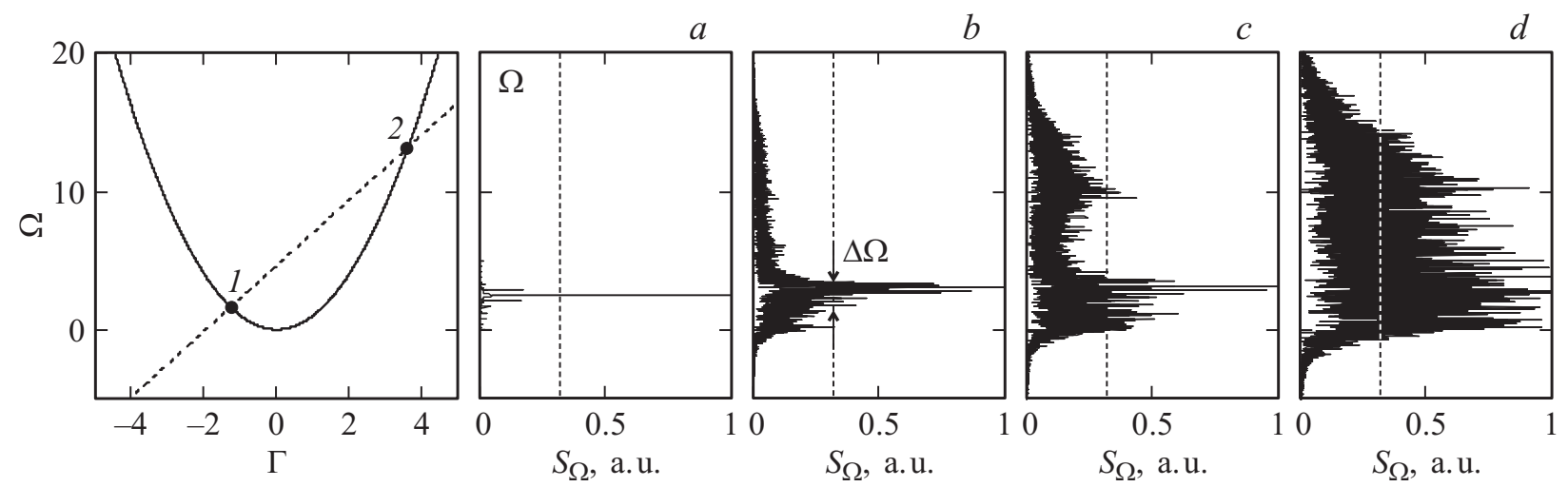

Рис. 3. Дисперсионные характеристики волны и пучка в режиме пересечения при $\Delta=-1.9$ (слева) и спектры выходного сигнала при увеличении параметра тока, $I_{0}: a-0.2, b-1.0, c-2.0, d-3.0$ (пунктиром обозначен уровень $-10 \mathrm{~dB}$ ).

Последняя соответствует прямой линии, наклон которой задается питч-фактором. Точки пересечения кривых (6), (7) на рис. 1 определяют низкочастотный (точка 1) и высокочастотный (точка 2) резонансы:

$$
\Omega_{1,2}=\frac{4}{g^{2}}\left(\frac{2}{g^{2}}-\Delta \mp \frac{2}{g} \sqrt{\frac{1}{g^{2}}-\Delta}\right) .
$$

Как будет показано ниже, частотный интервал между указанными резонансами

$$
\Delta \Omega^{*}=\Omega_{2}-\Omega_{1}=\frac{16}{g^{3}} \sqrt{\frac{1}{g^{2}}-\Delta}
$$

фактически определяет максимальную ширину спектра генерации при больших превышениях над порогом.

Здесь следует еще раз отметить, что описать наклон дисперсионной характеристики электронного пучка (7) и корректно определить резонансные частоты $\Omega_{1,2}$ позволяет учет временной производной в уравнениях движения электронов (3). В пренебрежении указанной производной дисперсионная характеристика пучка представляет собой вертикальную линию с единственным пересечением с дисперсионной характеристикой волны.
Для исследуемой задачи многочастной генерации это приводит к значительным отличиям в спектре генерации гиротрона при больших превышениях над порогом.

\section{2. Результаты моделирования}

Дальнейшее исследование динамики процессов в гиротроне проводилось путем численного моделирования самосогласованной системы уравнений (2), (3) при фиксированной длине резонатора $L=15$ и значении питч-фактора $g=1.3$, которое является типичным для современных гиротронов.

Отметим, что при учете наклона дисперсионной характеристики пучка единственная точка синхронизма имеет место только в режиме касания, когда электронный пучок взаимодействует с попутной волной. При выбранных параметрах этому случаю соответствует значение расстройки $\Delta=0.6$. Как показывает моделирование, в этих условиях при увеличении параметра тока ширина спектра хаотической генерации изменяется незначительно (рис. 2).

В то же время при $\Delta<0$, когда электронный пучок находится в синхронизме одновременно с обратной и 


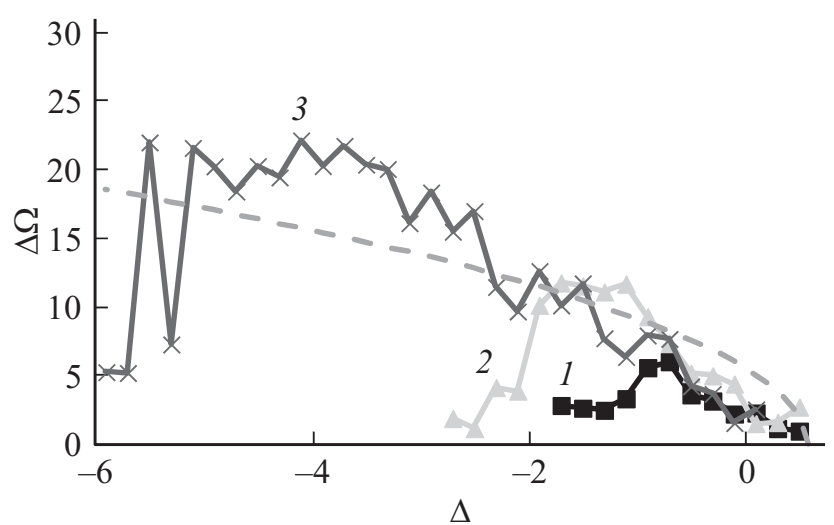

Рис. 4. Зависимость ширины спектра излучения по уровню $-10 \mathrm{~dB}$ от параметра $\Delta$ при различных значениях параметра тока, $I_{0}: 1-1.0,2-2.0,3-10.0$. Штриховая линия соответствует разности резонансных частот в точках 1 и 2 , определяемой формулой (9).

попутной волнами, при росте превышения над порогом происходит существенное расширение спектра генерации (рис. 3). При этом сначала реализуется хаотическая генерация со спектром, сосредоточенным вблизи низкочастотного резонанса $\Omega_{1}$ (рис. $3, a$ ), а затем по мере роста параметра тока $I_{0}$ шумовой пьедестал заполняет интервал частот между низкочастотным и высокочастотным резонансами (рис. $3, b$ ). Начиная с некоторого значения $I_{0}$, появляется локальный спектральный максимум в окрестности высокочастотного резонанса $\Omega_{2}$ (рис. $3, c$ ) Далее имеет место расширение спектра относительно $\Omega_{2}$ как в область более высоких, так и в область более низких частот. В конечном итоге происходит перекрытие полос хаотической генерации, и имеет место формирование шумового сигнала с практически равномерным спектром (рис. 3, $d$ ), ширина которого $\Delta \Omega$ близка к разности частот синхронизма в точках 1 и 2 , определяемых формулой (9).

На рис. 4 показаны зависимости ширины спектра хаотической генерации $\Delta \Omega$ от величины расстройки синхронизма при различных значениях параметра тока. Видно, что ширина спектра, близкая к $\Delta \Omega^{*}$, достигается при параметре тока $I_{0} \geq 2$. Отметим, что при заданном параметре тока существует предельное значение параметра расстройки $\Delta$, при котором фактически происходит срыв хаотической генерации в высокочастотной области, в результате чего ширина спектра резко уменьшается. В свою очередь, для достижения максимальной ширины спектра по мере роста тока следует уменьшать значение параметра расстройки синхронизма.

На рис. 5 показаны зависимости выходной мощности гиротрона $P=\operatorname{Im}\left(a \partial a^{*} / \partial Z\right)$ от времени в режиме двойного пересечения при параметрах тока $I_{0}$, соответствующих рис. 3. Видно, что по мере увеличения $I_{0}$ выходной сигнал трансформируется от квазипериодического (рис. 5, $a$ ) к хаотическому (рис. 5, $b-e$ ). При этом следует обратить внимание, что в исследуемых условиях излучение в режиме хаотической генерации представляет собой случайную последовательность коротких импульсов, длительность которых обратно пропорциональна ширине спектра, определяемой формулой (9). При этом пиковая мощность $P_{\text {peak }}$ таких импульсов может существенно превосходить средний по времени уровень излучения $\bar{P}$. Так, если вблизи границы области хаотической генерации $\left(I_{0}=0.5\right)$ отношение пиковой мощности импульсов к средней мощности излучения составляет $P_{\text {peak }} / \bar{P} \approx 6-7$ (рис. $\left.5, b\right)$, то при $I_{0}=3$ данное
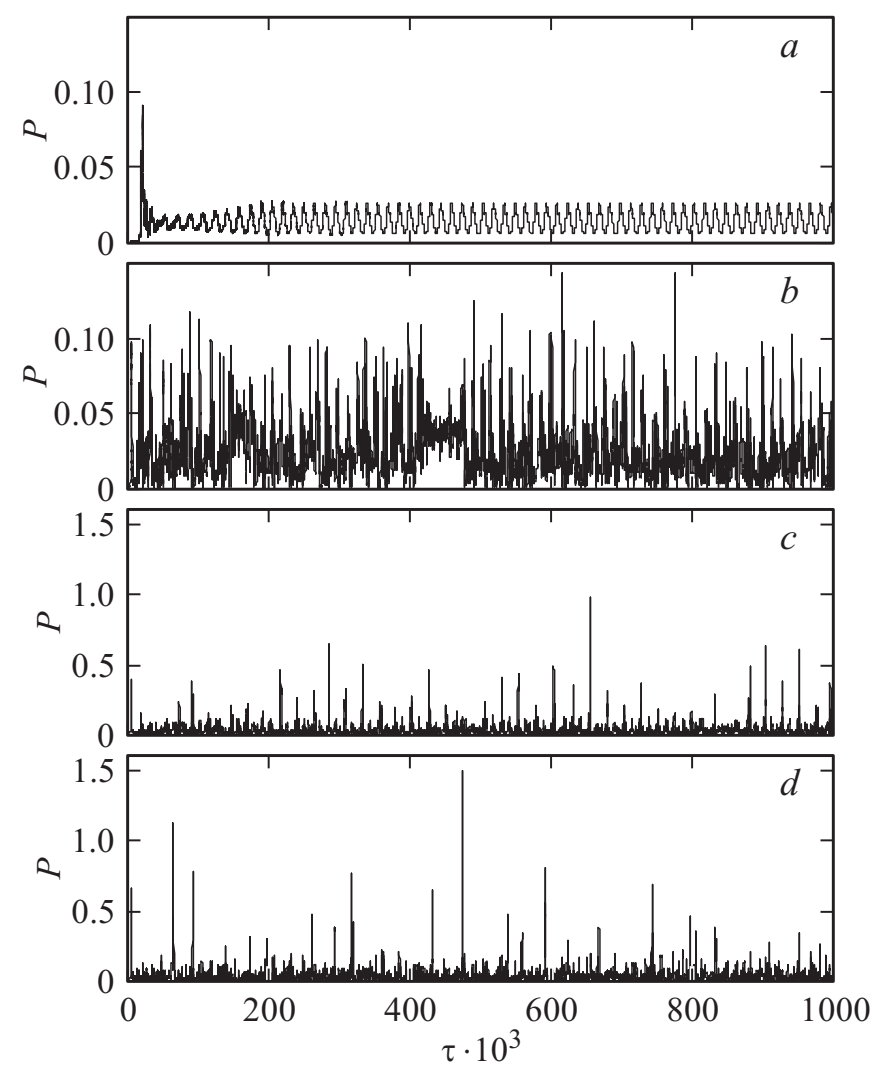

Рис. 5. Временнб́е зависимости мощности выходного излучения гиротрона в режиме пересечения дисперсионных характеристик $(\Delta=-1.9)$ при различных превышениях над порогом, $I_{0}: a-0.2, b-1.0, c-2.0, d-3.0$.

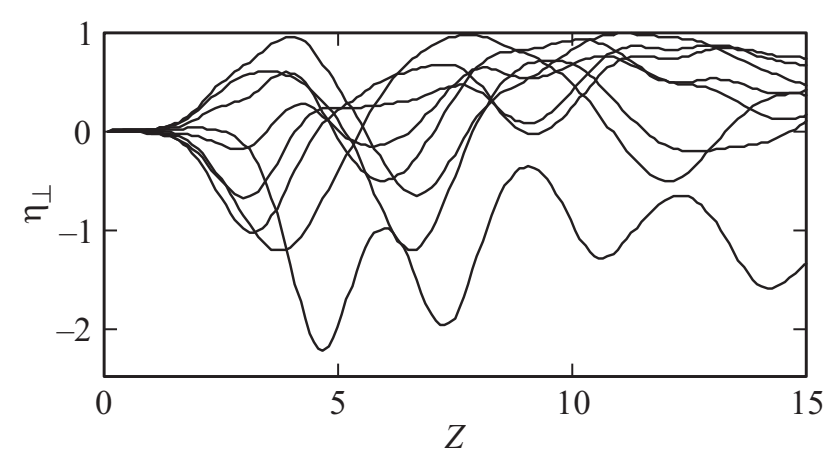

Рис. 6. Типичные зависимости изменения поперечного КПД отдельных электронов с различными фазами влета от продольной координаты $\left(I_{0}=3.0, \Delta=-1.9, \tau=200\right)$. 

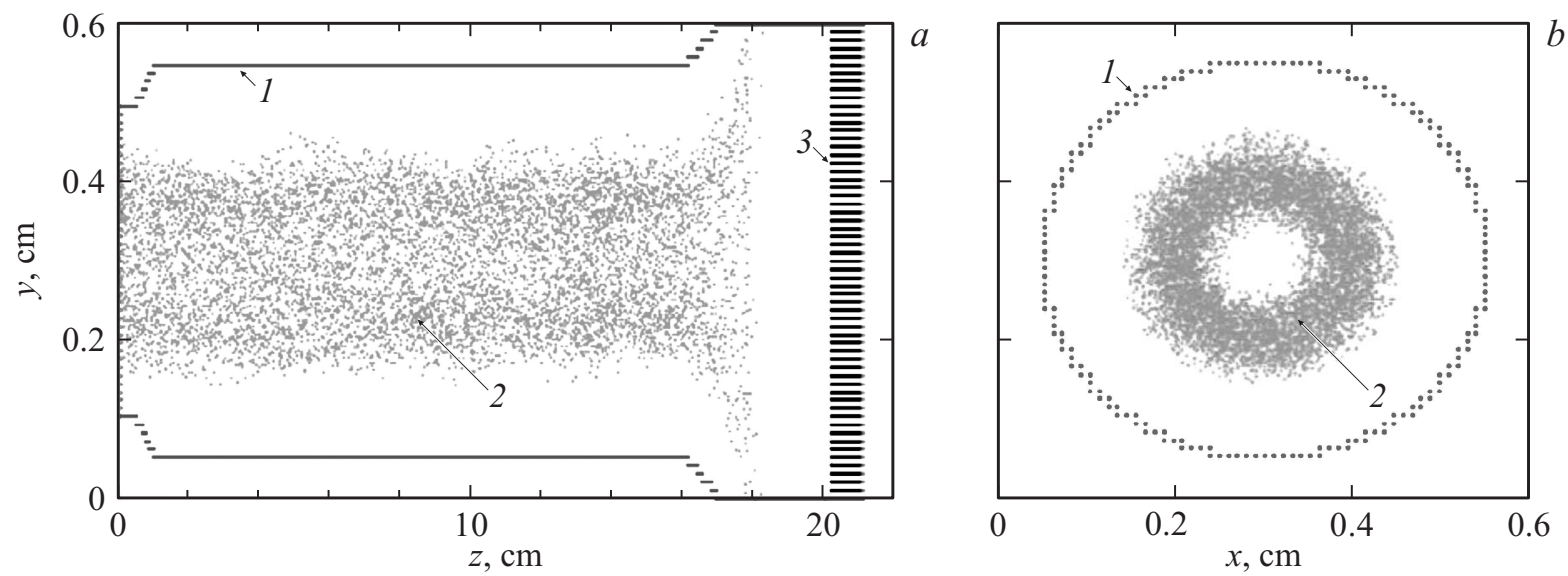

Рис. 7. Геометрия пространства взаимодействия гиротрона, используемая в трехмерном РІС-моделировании: $1-$ резонатор, $2-$ винтовой электронный пучок, 3 - поглощающий слой, имитирующий условия излучения на коллекторном конце пространства взаимодействия. Проведены продольное $(a)$ и поперечное $(b)$ сечения резонатора, а также мгновенное положение макрочастиц.
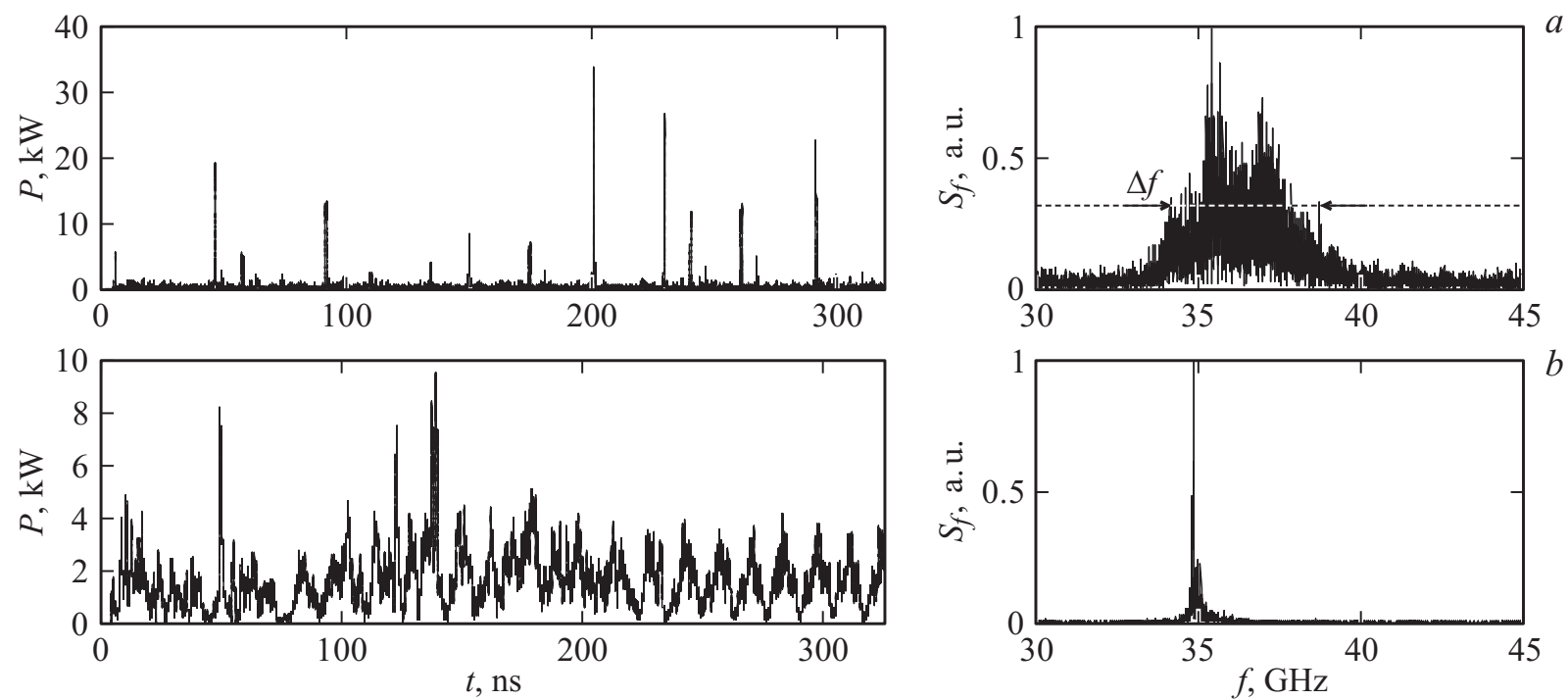

Рис. 8. Зависимости выходной мощности от времени и спектр излучения в трехмерном РІС-моделировании: $a-$ в режиме пересечения дисперсионных характеристик $(H=13.2 \mathrm{kOe}) ; b-$ в режиме касания дисперсионных характеристик $(H=12.45 \mathrm{kOe})$.

отношение возрастает до значений 40-45 (рис. 5,e). Следует отметить, что частота возникновения таких „гигантских“ импульсов относительно невелика.

Представляется, что подобные случайно возникающие импульсы, амплитуда которых более чем на порядок превышает фоновый уровень генерации, могут быть отнесены к классу так называемых „волн-убийц“ (freak/rogue waves). Это явление было первоначально описано в гидродинамике [21]. В настоящее время в ряде публикаций, в том числе экспериментальных, показано, что подобные эффекты в условиях развитой турбулентности могут наблюдаться в динамических системах различной физической природы [22].

Оценим возможность экспериментальной реализации режимов широкополосной хаотической генерации в миллиметровом диапазоне длин волн. Будем считать, что параметры электронного пучка близки к используемым для запитки технологического гиротрона с рабочей частотой $35 \mathrm{GHz}$, работающего на второй гармонике гирочастоты [23]: энергия электронов $20 \mathrm{keV}$, ток $2 \mathrm{~A}$, питч-фактор 1.3. Однако в отличие от стандартного режима генерации в данном гиротроне предположим, что взаимодействие с рабочей $T E_{1,1}$-волной организуется на первой гармонике гирочастоты. Тем самым при указанных выше токах пучка обеспечивается необходимое для реализации хаотических режимов превышение над порогом. Естественно, что при центральной частоте генерации $\sim 35 \mathrm{GHz}$ такой переход требует соответствующего увеличения напряженности ведущего магнитного поля.

Пусть рабочее пространство формируется цилиндрическим волноводом с радиусом $2.5 \mathrm{~mm}$ (критическая ча- 
стота низшей $T E_{1,1}$-моды при этом составляет $35.1 \mathrm{GHz}$ ) и длиной $15 \mathrm{~cm}$. Выбранным физическим параметрам соответствует значение параметра тока $I_{0} \approx 3$ и нормированная длина резонатора $L \approx 15$. Согласно результатам моделирования (рис. $3, d$ ) в этом случае относительная ширина спектра хаотической генерации при средней мощности излучения $600-800 \mathrm{~W}$ может достигать $\sim 15 \%$, что соответствует абсолютному значению $\sim 5 \mathrm{GHz}$. Следует отметить, что для исследованных режимов характерен достаточно низкий средний электронный КПД на уровне нескольких процентов. Это связано с тем, что движение электронов в подобных режимах носит хаотический характер, при котором отдельный электрон при пролете пространства взаимодействия многократно переходит из тормозящей в ускоряющую фазу поля и наоборот. В результате энергия отдельных электронов может достаточно сильно меняться, что иллюстрирует рис. 6 , где показаны мгновенные зависимости КПД отдельных электронов от продольной координаты. Таким образом, имеет место значительное расширение функции распределения электронов на выходе из рабочего пространства как в сторону меньших, так и больших энергий. Однако среднее изменение энергии электронов, которое и определяет электронный КПД, оказывается достаточно малым.

\section{3. Моделирование режимов широкополосной генерации В гиротронах на основе трехмерного PIC-кода KARAT}

Проведенный на основе усредненных уравнений (2), (3) анализ демонстрирует возможность реализации режимов сверхширокополосной генерации в гиротронах. Вместе с тем полученные результаты требуют подтверждения в рамках альтернативных методик, в частности, в связи с вопросом о применимости метода медленно меняющихся амплитуд для описания короткоимпульсных режимов генерации. С этой целью проведено прямое численное моделирование методом крупных частиц (particle-in-cell) на основе трехмерной версии PIC-кода KARAT.

Используемая в РIC-моделировании геометрия пространства взаимодействия представлена на рис. 7. Помимо регулярного участка с параметрами, указанными в разд. 2, резонатор гиротрона включал закритическое сужение на катодном и расширение на коллекторном конце пространства взаимодействия. Кроме того, был принят во внимание начальный разброс электронов по поперечным скоростям на уровне $20 \%$. После окончания взаимодействия электроны высаживались на стенку электродинамической системы за счет введения спадающего участка магнитного поля. Для моделирования условий излучения на коллекторном конце пространства взаимодействия был размещен поглощающий слой с переменной проводимостью, коэффициент отражения от

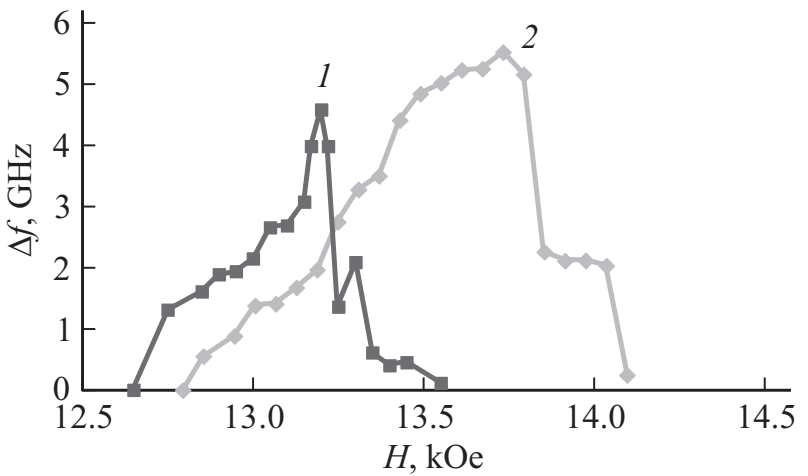

Рис. 9. Сравнение зависимостей ширины спектра хаотической генерации от магнитного поля, полученных на основе трехмерного PIC-моделирования (1) и усредненных уравнений (2).

которого не превышал $1 \%$ от мощности падающего излучения. Число узлов сетки составляло $\sim 2.4 \cdot 10^{5}$, число макрочастиц в пространстве взаимодействия $\sim 3 \cdot 10^{4}$. Отметим, что при увеличении числа узлов и количества частиц существенные отличия в динамике системы не возникали.

Проведенное моделирование подтвердило возможность существенного расширения полосы хаотической генерации в гиротронах. В режиме пересечения дисперсионных характеристик максимальная ширина спектра на уровне $-10 \mathrm{~dB}$ достигала $\sim 4.5 \mathrm{GHz}$ (рис. $8, a$ ), в то время как при касании дисперсионных характеристик аналогичное значение не превышало $100 \mathrm{MHz}$ (рис. 8, $b$ ). Средняя мощность широкополосного излучения составляла $460 \mathrm{~W}$, что соответствует КПД $\sim 1.2 \%$. Выходной сигнал в РІС-моделировании представлял собой хаотическую последовательность коротких $(0.2-0.5 \mathrm{~ns})$ импульсов большой мощности - „волн-убийц“. Отношение пиковой мощности таких импульсов к средней мощности излучения достигало $\sim 70$. Расхождение этого значения с результатами, полученными выше на основе усредненных уравнений, связано с тем, что адекватное описание формирования „волн-убийц“ в гиротроне требует построения модели, учитывающей изменение продольного импульса электронов. Необходимость такого учета обусловлена короткой длительностью „волн-убийц“, в результате чего на их фронтах возникают значительные перепады электрического поля. Соответственно инициируются сильные поперечные магнитные поля, которые, в свою очередь, приводят к значительному изменению продольного импульса частиц.

Сравнение зависимостей ширины спектра от магнитного поля, полученных на основе РІС-моделирования и на основе моделирования усредненных уравнений (2), (3), показало хорошее соответствие (рис. 9). Отличие в значениях магнитных полей, при которых достигается максимум ширины спектра, можно объяснить дискретностью задания стенок круглого волновода в прямоугольной сетке, вследствие чего происходит увеличение (до 4\%) эффективного радиуса волновода относительно заданного. 


\section{Заключение}

Проведенный анализ демонстрирует возможность реализации режимов сверхширокополосной генерации в гиротронах с большой надкритичностью. Существенное расширение полосы генерации достигается в области значений ведущего магнитного поля, при которых имеет место одновременное взаимодействие с обратной и попутной волнами, точки резонанса с которыми существенно разнесены по частоте. Важно отметить, что спектр излучения при этом существенно более равномерный по сравнению, например, с генератором на основе гиро-ЛБВ с запаздывающей обратной связью [24], где в спектре сигнала четко идентифицируются компоненты „холодных“ мод с относительно низким шумовым пьедесталом.

На основе проведенного моделирования показано, что для параметров электронных пучков, реализуемых на основе существующих гиротронных стендов, относительная ширина спектра шумового излучения миллиметрового диапазона может достигать порядка $10 \%$. Низкие значения среднего КПД гиротронов в исследуемом режиме (на уровне $1-2 \%$ ) делают целесообразным их использование в качестве задающих генераторов шумовых сигналов. Далее такие сигналы могут быть усилены, например, в гиро-ЛБВ с винтовым гофрированным волноводом, обладающей близкой по значению полосой усиления [25], с уровнем выходной мощности десятки и сотни киловатт.

Работа выполнена при поддержке Российского научного фонда, грант № 16-42-01078.

\section{Список литературы}

[1] Глявин М.Ю., Денисов Г.Г., Запевалов В.Е., Кубтин А.Н., Лучинин А.Г., Мануилов В.Н., Морозкин М.В., Седов А.С., Чирков А.B. // Радиотехника и электроника. 2014. Т. 59. № 8. C. 745-751.

[2] Alberti S., Braunmueller F., Tran T.M., Hogge J.-P., Genoud J., Tran M.Q. // Terahertz Sci. Tech. 2014. Vol. 7. N 1. P. 23-38.

[3] Thumm M. // IEEE T. Plasm. Sci. 2014. Vol. 42. N 3. P. 590 599.

[4] Puri S. // Plasm. Phys. 1974. Vol. 16. P. 517-526.

[5] Anza S., Vicente C., Gil J., Mattes M., Wolk D., Wochner U., Boria V.E., Gimeno B., Raboso D. // IEEE T. Microw. Theory 2012. Vol. 60. N 7. P. 2093-2105.

[6] Kulpa K. Signal Processing in Noise Waveform Radar. Boston, London: Artech House, 2013. 248 p.

[7] Ginzburg N.S., Nusinovich G.S., Zavolsky N.A. // Int. J. Electron. 1986. Vol. 61. N 6. P. 881-894.

[8] Chang T.H., Chen S.H., Barnett L.R., Chu K.R. // Phys. Rev. Lett. 2001. Vol. 87. N 6. P. 064802.

[9] Ronald K., Cross A.W., Phelps A.D.R., He W. // J. Phys. D: Appl. Phys. 2001. Vol. 34. P. L17-L22.

[10] Alberti S., Ansermet J.-Ph., Avramides K.A., Braunmueller F., Cuanillon P., Dubray J., Fasel D., Hogge J.-Ph., Macor A., Rijk E., Silva M., Tran M.Q., Tran T.M., Vuillemin Q. // Phys. Plasmas. 2012. Vol. 19. P. 123102.
[11] Глявин М.Ю., Запевалов В.Е., Куфтин А.Н., Лучинин А.Г. // Изв. вузов. Радиофизика. 2000. Т. 43. № 5. C. $440-444$.

[12] Rozental R.M., Zaitsev N.I., Kulagin I.S., Ilyakov E.V., Ginzburg N.S. // IEEE T. Plasm. Sci. 2004. Vol. 32. N 2. P. 418-421.

[13] Rozental R.M., Ginzburg N.S., Glyavin M.Yu., Sergeev A.S. // IEEE T. Microw. Theory. 2006. Vol. 54. N 6. P. 2741-2744.

[14] Рекомендации МСЭ-R SM.1755 (05/06). Характеристики сверхширокополосной технологии.

[15] IEEE Std 1672-2006. IEEE Standard for Ultrawideband Radar Definitions. 4 May 2007.

[16] Ginzburg N.S., Sergeev A.S., Zotova I.V. // Phys. Plasm. 2015. Vol. 22. P. 033101.

[17] Airila M.I., Dumbrajs O. // Nucl. Fusion. 2003. Vol. 43. P. 1446-1453.

[18] Nusinovich G.S. Introduction to the physics of gyrotrons. Baltimore and London: The John Hopkins University Press, 2004. 335 p.

[19] Blokhina E.V., Kuznetsov S.P., Rozhnev A.G. // IEEE T. Electron Dev. 2007. Vol. 54. N 2. P. 188-193.

[20] Тараканов В.П. В кн.: Математическое моделирование. Проблемы и результаты. М.: Наука, 2003. С. 456.

[21] Extreme Ocean Waves / Ed. by E. Pelinovsky, C. Kharif. Springer Science + Business Media B.V., 2008. 196 p.

[22] Onorato M., Residori S., Bortolozzo U., Montinad A., Arecchi F.T. // Phys. Rep. 2013. Vol. 528. P. 47-89.

[23] Bykov Yu., Eremeev A., Glyavin M., Kholoptsev V., Luchinin A., Plotnikov I., Denisov G., Bogdashev A., Kalynova G., Semenov V., Zharova N. // IEEE T. Plasm. Sci. 2004. Vol. 32. N 1. P. 67-72.

[24] Ginzburg N.S., Rozental R.M., Sergeev A.S., Zotova I.V., Tarakanov V.P. // Phys. Plasm. 2016. Vol. 23. P. 103106.

[25] Samsonov S.V., Gachev I.G., Denisov G.G., Bogdashov A.A., Mishakin S.V., Fiks A.S., Soluyanova E.A., Tai E.M., Dominyuk Y.V., Levitan B.A., Murzin V.N. // IEEE T. Electron Dev. 2014. Vol. 61. N 12. P. 4264-4267. 